\title{
Comparative Study of Terrestrial Birds in the Disturbed and Undisturbed Vegetation Types of Ikere Forest Reserve, Ekiti State, Nigeria
}

\section{Ogunyemi 00*}

Department of Forest Resources and Wildlife management, Ekiti State University, Nigeria

*Corresponding author: Ogunyemi 00, Department of Forest Resources and Wildlife management, Ekiti State University, Ado-Ekiti, Nigeria, Email: olumideogunyemi80@yahoo. com

\section{Review Article \\ Volume 4 Issue 4}

Received Date: March 23, 2020

Published Date: May 21, 2020

DOI: $10.23880 /$ jenr-16000201

\section{Abstract}

Birds are an important component of earth's ecosystems. The primary objective of the study was to assess the effect of disturbance on bird species diversity and abundance in the two stratified habitat types (disturbed and undisturbed) of Ikere Forest Reserve from January to December, 2017. Data were collected by employing transect count technique in the early morning (6.00-10.00a.m) and late afternoon (4.00-6.00 p.m) for four consecutive days every month in the two vegetation types. During the study, a total of 551 individual birds comprising of 59 species, 18 families and 11 Orders were recorded. Out of 59 identified species of birds, 46 (78\%) of the species were recorded from disturbed vegetation, while 30 species were recorded in the undisturbed vegetation. Order Passeriformes constituted the numerically dominant Order represented with 16 species while Pelecaniformes, Galliformes and Colliformes were the least dominant Orders represented with one species each. At the family level, family Nectariniidae was numerically the dominant family represented with 9 species. At species level, Rufous-backed manikin Louchura nigriceps and Great-white egret Egretta alba were numerically the most dominant species. The total abundance of birds was significantly different $(\mathrm{P}>0.05)$ among the two vegetation types. The total abundance of birds ranked higher with 393 birds in the disturbed vegetation. The Shannon- Wiener index of diversity (H') varied from 3.08 in undisturbed vegetation to 3.61 in the disturbed vegetation. There were significant differences in the bird species richness that the different vegetation types supported. The results indicate that disturbed vegetation supported a higher diversity and abundance of birds than the undisturbed vegetation.

Keywords: Species Diversity; Vegetation; Transect; Abundance

\section{Introduction}

Nigeria has some of the most beautiful ecosystems which accommodates 850 species of resident birds of these 640 species are known to be breeding residents while a further group of 40 species are rare [1]. The biological diversity of birds in Nigeria is associated with unique geological history, diverse climatic and physical conditions of the country [2].
The vegetation range of Nigeria has distinctive differences because it is such a large country, stretches from the beaches and mangrove swamps of the Gulf of Guinea through the remnants of the rainforest to the Savannas and thorn Scrub of the north. These agro-ecological diversity and niches which have their own peculiarity in climatic conditions provides habitat for fascinating variety of birds. Birds are an important component of many ecosystems; such 
that their study can alert us to what is going wrong in the environment, as birds are excellent environmental indicator [3]. Tesfahunegny [4] described birds as agents of nutrient cycles, plant gene flow through pollination, seed dispersal, controls population size of harmful insects, environmental sanitation through scavenging of carrion. Rands, et al. [5] opined that the probable cause of the avian decline are multifaceted and involve various complex combinations of obvious ones such as habitat destruction, habitat fragmentation or loss, pollination and over harvesting. Tropical rainforest accommodates the most diverse range of flora and fauna in the world. The natural resources of tropical rainforest are presently and extensively being utilized for economic purpose. In Nigeria, deforestation has led to the loss of habitats of many biodiversity including forest birds which depend on a healthy forest ecosystem. The importance of forest ecosystem cannot be overemphasized as increase in complexity of vegetation structure, floristic composition and heterogeneity engenders increase in diversity of bird species [6]. Any form of disturbance, natural or artificial destabilizes vegetation structure and bird community structure [7]. The alteration in the habitat structure invariably affect negatively the essential requirements of birds especially the nest, cover and food. Human induced disturbance can have a significant negative effect on breeding success by causing nest abandonment at the same time increase vulnerability to predation. In recent years the interest in conservation of birds had grown in many countries and regions emphasized the need for more studies of the ecological impact of vegetation disturbance on bird species. Sethy [8] asserted that assessment of bird community is important and needed for monitoring changes, which will lead to efficient and effective conservation of biodiversity. Having knowledge on biodiversity and composition of bird communities' as a function of habitat variation is crucial to determine the health status of the local ecosystem. In the recent times Ikere Forest Reserve vegetation community structure disruption has taken a higher trend due to high frequency of logging activities as a result of high rate of human population growth. It is therefore necessary to understand the effect of habitat disturbance on bird species community structure in order to know the effects of disturbed and undisturbed ecosystems on birds. This will definitely play a dominant role in prioritize future conservation of bird species in the reserve.

\section{Methodology}

\section{Description of the Study Area}

The study was carried out in the $72.52 \mathrm{~km}^{2}$ area of Ikere Forest Reserve in Ekiti State, Nigeria. The Ikere Forest Reserve is located between $70321 \mathrm{~N}$ and $50211431 \mathrm{E}$. The prevailing climate is tropical with an average temperature of $25^{\circ} \mathrm{C}$ all year round and high relative humidity. The rainy season has an average of 240 days with mean annual rainfall of 1250 to $1400 \mathrm{~mm}$. The pattern of rainfall distribution over the long rainy season between April and mid-November is bimodal with a peak in September while the dry season stretches from mid-November to the end of March. The terrain of the reserve is flat to slightly undulating with elevation of between 12 to $110 \mathrm{~m}$ above sea level. Light South westerly and North easterly winds blow over the area throughout the year which characterized the seasons in the reserve into rainy and the dry season. The reserve is surrounded by villages and farm settlements inhabited by the largely agrarian population that engages in small holder food and tree cash crop production systems with poor farming methods. The study area has a diversified woody plant species. The dominant tree species include Dacroydes edulis, Chrysophyllum albidum, Afzelia Africana, Cola edulis, Garcinia kola, Cola acuminate, Gmelina arborea, Pterocarpus osun, Gliricidia sepium, Parkia biglobosa, Mansonia altissima and Baillaonella toxisperma. Also in certain area of study the vegetation is characterized by secondary forests especially where the vegetation types is accentuated by farming activities, where yam, cocoyam, cassava and maize are grown as major crops. The major challenges in Ikere Forest Reserve leading to degradation of the environment are the expansion of farmland at the expense of forestland, extensive logging, burning and high growing demand for fire wood due to ever increasing human population. Burning of vegetation cover has led to frequent destruction of the fragile forest ecosystem in the reserve. Several forest patches show sign of fire damage with some trees badly scorched and the under storey destroyed. In the some places, the vegetation have been cut and burn for farming posing severe danger to the future of the unique fauna of the habitat. Logging activities is also widespread in the reserve. Within this landscapes, study area was stratified into two main habitats named as disturbed (D1) and undisturbed (D2) forests based on level of disturbance and proximity to human settlements.

\section{Survey of Avifauna}

The census method was used to survey the abundance and diversity of avifauna species of Ikere Forest Reserve, Ekiti State. The survey was conducted on monthly basis from January to December, 2017 in 12 plots of approximately 50 hectares (ha) in each of the two stratified habitat types: disturbed and undisturbed habitats in Ikere Forest Reserve, using the transect count method described by Bibby [9]. The relative value of each habitat for attracting different bird species was determined by establishment of one transect of 1 kilometer $(\mathrm{km})$ length in each of the 12 plots. The birds were observed by walking along the established transects in each of the habitat types for four consecutive days every month. Data collection commenced about 30 minutes after dawn and was carried out for five hours daily from 6:30- 


\section{Journal of Ecology and Natural Resources}

10:00am in the morning and 4:30 -6:00pm in the evening to coincide with time the activities of birds were prominent [10]. To avoid effects of pseudo-replication in the counting of birds, transect were reasonably space out by $300 \mathrm{~m}$ distance apart [11].

In all transects, a record was made of all the types and group number of bird species through direct observation. The birds were observed with the aid of binoculars (Olympus 10 and 42) to the species level and their taxonomic groups were properly categorized based on field guide to birds of Western Africa.

\section{Data Analysis}

Data were arranged, organized and entered into Microsoft excel or analysis. The cumulative list of bird species recorded in each of the two habitat types was used as a basic measure of avian richness. The relative abundance of avian species was determined using the encounter rate that gives crude ordinal scales of abundance (abundant, common, frequent, uncommon and rare) Bibby [9] Encounter rate incorporates the field hours of observation and the number of individuals of each species observed. This allows encounter rate to be calculated for each species by dividing the number of birds recorded by the number of hours spent searching, giving a figure of birds per hour for each species. The abundance categories have the following abundance scores $\leq \leq 1.0$ (rare), 1.1 - 2.02 (uncommon), 2.1 - 10 (frequent), 10.1 40.0 (common) and $\geq 40$ (abundant) was employed. Birds' diversity was calculated using Shannon-Weiner diversity index $\left(\mathrm{H}^{\prime}\right)$ which was calculated using formula:

$$
\begin{gathered}
\text { Shannon -Wiener index of diversity }\left(\mathrm{H}^{\prime}\right) \\
\qquad \mathrm{H}^{1}=-\sum \mathrm{p}_{\mathrm{i}} \log \mathrm{pi}
\end{gathered}
$$

Where $\mathrm{P}_{1}$ is the proportion of ith species to total abundance value

Species Richness index (D) was calculated by with the following equation

$$
\mathrm{D}=\frac{\mathrm{S}-1}{\ln \mathrm{N}}
$$

Where $\mathrm{D}=$ Species Richness Index, $\mathrm{S}=$ Total number of species and $\mathrm{N}=$ Total number of individuals

To understand bird community similarity among habitats, Sorensen's coefficient was applied to determine the similarities between the two habitats. It was calculated as:

$$
\mathrm{C}=\frac{2 \mathrm{WX} 100}{\mathrm{~A}+\mathrm{B}}
$$

Where $\mathrm{C}=$ index of similarity

$\mathrm{W}=$ No of species common to both sample

$A=$ No of species in sample $A$

$B=$ No of species in sample $B$
To test for variation among bird species composition in the two habitats. One way analysis of variance (ANOVA) was employed using SPSS version16 computer software to statistically test for variation among bird species richness, Shannon-Wiener diversity index, bird species abundance and bird feeding guilds in the disturbed and undisturbed habitats. Graphs were drawn by using Microsoft offices excel 2007 computer software.

\section{Results}

\section{Species composition of birds}

The result of this study showed that a total of 546 birds belonging to 59 species, 18 families and 11 orders inhabit the disturbed and undisturbed vegetation of Ikere Forest Reserve, Ekiti State. Order Passerriformes constituted the numerically dominant order in the disturbed vegetation representing $32.6 \%$ of the species $(n=15)$ while Pelecaniformes and Anseriformes were the least dominant orders represented with 1 and 2 species respectively (Table 1). In the undisturbed habitat the order Falconiformes was the numerically dominant order represented with 7 species which accounts $23.3 \%$ of the identified species in the undisturbed habitat while Galliformes and Colliformes were the least dominant orders represented with 1 specie each (Table 2). At the family level, family Nectariniidae had the largest number of species represented with 9 species which accounts $15.25 \%$ of the identified species in the disturbed vegetation; while in the undisturbed vegetation, the family Accipitridae was numerically the dominant family represented with 7 species which account $11.86 \%$ of the observed species (Tables 1 and 2).

At species level, Rufous-backed manikin (Lonchura nigriceps) and Great white egret (Egretta alba) were numerically the dominant species recorded in the disturbed vegetation of the reserve with 30 number of individuals each contributing $15.46 \%$ of the total bird abundance in the disturbed vegetation while Augur buzzard (Buteo rufofuscus) and Black kite (Mulvus migrans) ranked dominant in the undisturbed vegetation of the reserve with 30 and 14 number of individuals respectively, contributing $27.85 \%$ of the total bird abundance of the undisturbed vegetation.

\section{Distribution and Abundance of Birds}

The result of this study revealed that out of the totality of 59 bird species recorded during the study period, highest number: 46 species were recorded from disturbed vegetation while 30 species were recorded in the undisturbed vegetation. $28.8 \%$ of birds are common to both disturbed and undisturbed vegetation with $67.5 \%(\mathrm{n}=27)$ as compared to $32.5 \%(\mathrm{n}=13)$ of exclusive bird species in 
the undisturbed vegetation (Figure 1). Moreover, from the total 551 birds counted during the study, 393 of them were recorded from the disturbed vegetation, 158 birds were observed in the undisturbed vegetation. The total abundance of birds showed significant difference among the two study vegetation $(\mathrm{P}>0.05)$.

\section{Species Diversity and Community Similarity}

The disturbed habitat of the study area had the higher values of species diversity $\left(\mathrm{H}^{\prime}=3.61\right)$ and species richness $\left(D^{\prime}=7.37\right)$ than undisturbed habitat. The overall community similarity of the two study sites was fairly average $(S=0.47)$ (Table 3).

\section{Relative Abundance of Bird Species}

In this study, the numbers of bird species in different relative abundance categories in the disturbed and undisturbed vegetation of Ikere Forest Reserve revealed that among the 59 bird species observed, 16, 25 of them were rare species followed by 22,3 uncommon species while 8,2 species were in frequent category respectively. The relative abundance findings shows that majority of the species had low population sizes and were grouped under rare and uncommon species. Only 10 species were grouped under frequent species because they had relatively greater number of individuals (Figure 2).

\begin{tabular}{|c|c|c|c|}
\hline S/N & Species & Order & Family \\
\hline 1 & Cape Teal Anas capensis & Anseriformes & Anatidae \\
\hline 2 & Red-billed duck Anas erythrohynchos & Anseriformes & Anatidae \\
\hline 3 & White -backed vulture Gyps bengalensis & Accipitriformes & Accipitridae \\
\hline 4 & Verreaux's Eagle Aqua Verreauxii & Accipitriformes & Accipitridae \\
\hline \multirow[t]{2}{*}{5} & Martial Eagle Pole Maetus bellicosus & Accipitriformes & Accipitridae \\
\hline & Lizard buzzard Kaupifalco monogrammicus & Accipitriformes & Accipitridae \\
\hline 7 & African fish Eagle Halieetus vocifer & Falconiformes & Accipitridae \\
\hline 8 & Black kite milvus migrans & Falconiformes & Accipitridae \\
\hline 9 & Crowned Eagle stephaoeatus caronatus & Falconiformes & Accipitridae \\
\hline 10 & Grasshopper buzzard Butastus rufipennis & Falconiformes & Accipitridae \\
\hline 11 & Red- necked spurfool Francolinus levcoscepus & Galliformes & Phasianidae \\
\hline 12 & Scaly francolin Francolinus squamatus & Galliformes & Phasianidae \\
\hline 13 & Helmeted guinea fowl Numida meleagris & Galliformes & Numididae \\
\hline 14 & Red-eyed dove Stretopelia semitorquata & Columbiforms & Columbidae \\
\hline 15 & Ring-necked dove Streptopelia capicola & Columbiforms & Columbidae \\
\hline 16 & Yellow -neck spur fowl Francolinus levcoscepnus & Galliformes & Phasianidae \\
\hline 17 & Green pigeon Treron treron olax & Columbiformes & Columbidae \\
\hline 18 & Laughing dove Streptopelia senegalensis & Colubiformes & Columbidae \\
\hline 19 & Tambourine dove Turtur tympanistria & Columbiformes & Columbidae \\
\hline 20 & Green pigeon Treon australis & Columbifromes & Columbidae \\
\hline 21 & Emerald -spotted wood dove Turtur chalcospilos & Columbiformes & Columbidae \\
\hline 22 & Speckled pigeon Columba guinea & Columbiformes & Columbidae \\
\hline 23 & Emerald cuckoo Chrysococcyx cupreus & Cuculiformes & Cuculidae \\
\hline 24 & Red-chested cuckoo Cuculus solitarius & Cuciliformes & Cuculidae \\
\hline 25 & White - browed coucal Centropus supercilious & Cuculiformes & Cuculidae \\
\hline 26 & Wattled black Hornbill Ceratogyma atrata & Coraciiformes & Bucerotidae \\
\hline 27 & White -crested Hornbill & Coraciiformes & Bucerotidae \\
\hline 28 & Grey Hornbill Tockus hemprichii & Coraciiformes & Bucerotidae \\
\hline 29 & Yellow -billed hornbill Tockus flavirostris & Coraciiforms & Bucerotidae \\
\hline 30 & Golden pipit Tmetothylacus tenellus & Passeriformes & Motacillidae \\
\hline
\end{tabular}




\begin{tabular}{|c|c|l|c|}
\hline 31 & Yellow -throated wNgclay macronyx croceus & Passeriformes & Motacillidae \\
\hline 32 & Singing cisticola Cisticola cantans & Passeriformes & Cisticolidae \\
\hline 33 & Crombec sylvcetta Brachyura & Passeriformes & Macrosphenidae \\
\hline 34 & Black and white manikin Lonchura poensis & Passeriformes & Estrildidae \\
\hline 35 & Bronze manikin Lonchura cucullata & Passeriformes & Estrildidae \\
\hline 36 & Hunter's sunbird Nectarinia hunter & Passeriformes & Nectariniidae \\
\hline 37 & Green throated sunbird Nectarinia rubescens & Passeriformes & Nectariniidae \\
\hline 38 & Olive-bellied sunbird Nectarinia chloropygius & Passeriformes & Nectariniidae \\
\hline 39 & Red-chested sunbird Nectarinia erythrocerca & Passeriformes & Nectariniidae \\
\hline 40 & Regal sunbird Nectarinia ronia & Passeriformes & Nectariniidae \\
\hline 41 & Scarlet-Tufted malachite sunbird Nectarinia Johnstoni & Passeriformes & Nectariniidae \\
\hline 42 & Malachite sunbird Nectarinia famosa & Passeriformes & Nectariniidae \\
\hline 43 & Purple-breasted sunbird Nectarinia purpure ventri & Passeriformes & Nectariniidae \\
\hline 44 & Shining sunbird Nectarinia habessinica & Passeriformes & Nectariniidae \\
\hline 45 & Great Egret Egretta alba & Pelecaniformes & Ardeidae \\
\hline 46 & Rufous-backed manikin Lonchura poensis & Passeriformes & Estrildidae \\
\hline
\end{tabular}

Table 1: Bird species composition of disturbed vegetation in Ikere Forest Reserve.

\begin{tabular}{|c|c|c|c|}
\hline $\mathbf{S} / \mathbf{N}$ & Species & Order & Family \\
\hline 1 & Goliath Heron Ardea goliath & Pelecanifomres & Ardeidaee \\
\hline 2 & Black-headed Heron Ardea melanocephala & Pelecanifomres & Ardaidae \\
\hline 3 & Grey Heron Ardae cineral & Pelecanifomres & Ardeidae \\
\hline 4 & Purple Heron Ardea purpurea & Pelecaniformes & Ardeidae \\
\hline 5 & Little bittern Arderailus sturmii & Pelecaniformes & Ardeidae \\
\hline 6 & Red - billed duck Anas erythrorhynchos & Anseriformes & Anatidae \\
\hline 7 & Cape teal Anas capensis & Anseriformes & Anatidae \\
\hline 8 & White - backed vulture Gyps bengalensis & Accipitriformes & Accipitridae \\
\hline 9 & Long crested eagleLophaetus occipitalis & Accipitriformes & Accipitridae \\
\hline 10 & Lammergeyer Gypaetus barbatus & Falconiformes & Accipitridae \\
\hline 11 & African fish eagle Lophaetus occipitalis & Falconiformes & Accipitridae \\
\hline 12 & Augur buzzard Buteo rufofuscus & Falconiformes & Accipitridae \\
\hline 13 & Black kite Milvus migrans & Falconiformes & Accipitridae \\
\hline 14 & African hobby Falw cuvieri & Falconiformes & Accipitridae \\
\hline 15 & Ring - necked dove Streptopelia capicola & Columbiformes & Columbidae \\
\hline 16 & Green pigeon Treron olax & Columbiformes & Columbidae \\
\hline 17 & Tambourine dove Turtur tympanistria & Columbiformes & Columbidae \\
\hline 18 & Green pigeon Treron australis & Columbiformes & Columbidae \\
\hline 19 & Speckled pigeon Columba guinea & Columbiformes & Columbidae \\
\hline 20 & Speckled mouse bird Colius striatus & Colliformes & Collidae \\
\hline 21 & Emerald cuckoo Chrysococcyx cupreus & Cuculiformes & Cuculidae \\
\hline 22 & $\begin{array}{l}\text { Red - chested cuckoo } \\
\text { Cuculus solitaries }\end{array}$ & Cuculiformes & Cuculidae \\
\hline 23 & White - browed coucal Centropus superciliosus & Cuculiformes & Cuculidae \\
\hline
\end{tabular}




\begin{tabular}{|c|c|c|c|}
\hline 24 & Great blue turaco Corythaeola cristata & Cuculiformes & Musophagidae \\
\hline 25 & Wattled black hornbill Ceratogyma atrata & Coraciiformes & Bucerotidae \\
\hline 26 & White - crested hornbill Tropicranus albocristatus & Coraciiformes & Bucerotidae \\
\hline 27 & Gray hornbill Tockus flavirostris & Coraciiformes & Bucerotidae \\
\hline 28 & Yellow - billed hornbill Tockus flavirostris & Coraciiformes & Bucerotidea \\
\hline 29 & Black hornbill Ceratoauma atrata & Coraciiformes & Bucerotidae \\
\hline 30 & Yellow - necked spurfowl Francolinus leucoscepnus & Galliformes & Phasianidae \\
\hline
\end{tabular}

Table 2: Bird species composition of undisturbed vegetation in Ikere Forest Reserve.

\begin{tabular}{|c|c|c|c|c|c|c|}
\hline Study sites & S & D & N & D' & J' $^{\prime}$ & H' $^{\prime}$ \\
\hline Disturbed & 0.47 & 0.033 & 393 & 7.37 & 0.82 & 3.61 \\
\hline Undisturbed & & 0.068 & 158 & 5.93 & 0.70 & 3.08 \\
\hline
\end{tabular}

Table 3: Diversity, evenness and species richness indexes of birds in the study sites.

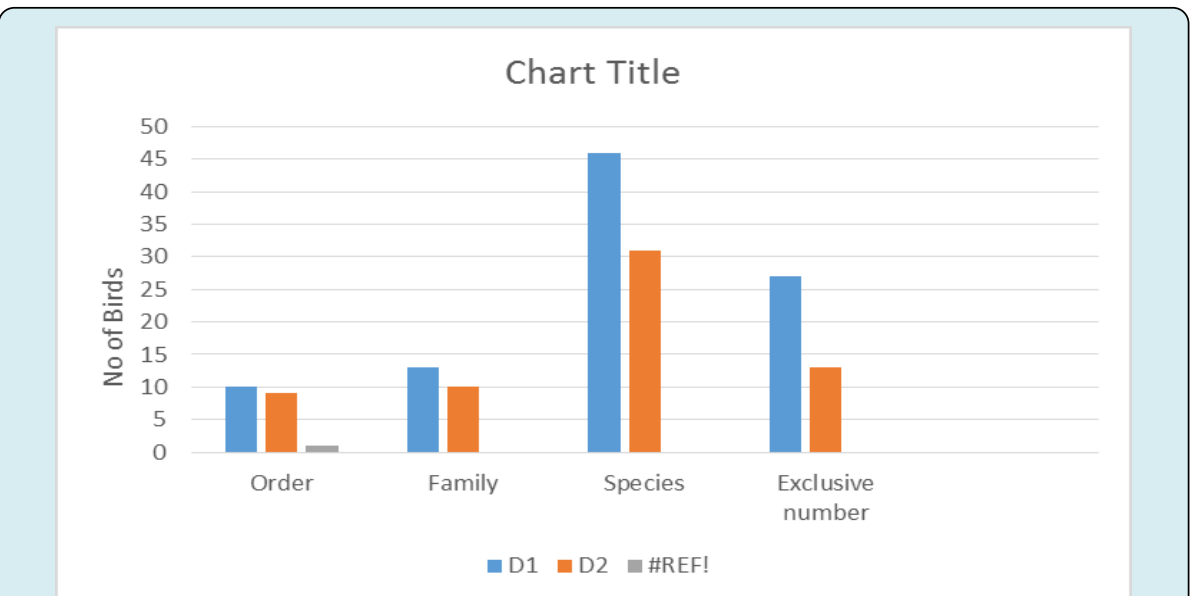

Figure 1: Table of order, family, species and exclusive species composition.

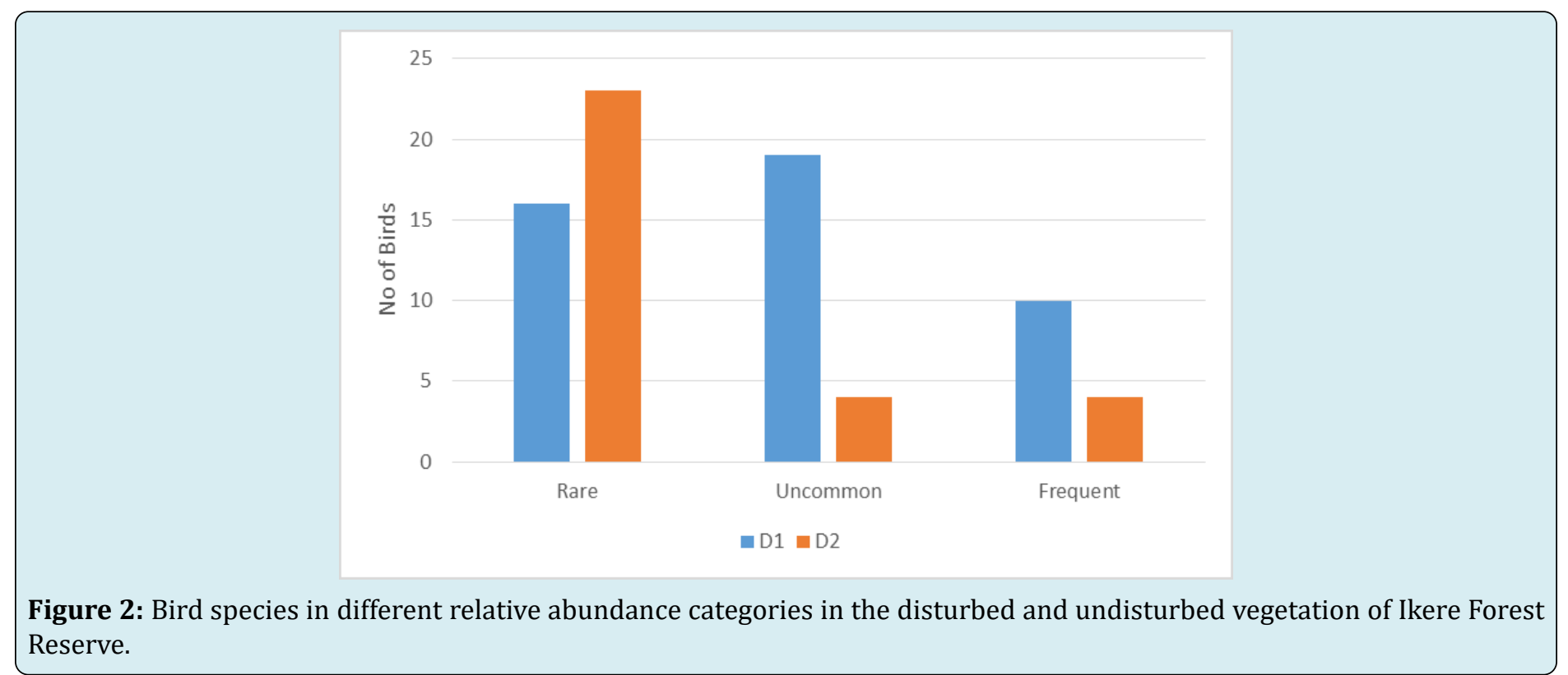




\section{Journal of Ecology and Natural Resources}

\begin{tabular}{|c|c|c|c|c|c|}
\hline \multirow{2}{*}{$\mathbf{S} / \mathbf{N}$} & \multirow{2}{*}{ Species } & \multirow{2}{*}{ Disturbed } & \multirow{2}{*}{ Undisturbed } & \multicolumn{2}{|c|}{ Ordinal scale } \\
\hline & & & & D1 & D2 \\
\hline 1. & $\begin{array}{l}\text { Goliath Heron } \\
\text { Ardea goliath }\end{array}$ & - & + & - & Rare \\
\hline 2. & $\begin{array}{l}\text { Black-headed Heron } \\
\text { Ardea melanocephala }\end{array}$ & $\therefore$ & + & - & Uncommon \\
\hline 3. & $\begin{array}{c}\text { Grey heron } \\
\text { Ardea cinerea }\end{array}$ & - & + & - & Rare \\
\hline 4. & $\begin{array}{l}\text { Purple Heron } \\
\text { Ardea purpurea }\end{array}$ & - & + & - & Rare \\
\hline 5. & $\begin{array}{c}\text { Cape Teal } \\
\text { Anas capensis }\end{array}$ & - & + & Uncommon & Rare \\
\hline 6. & $\begin{array}{c}\text { Red- billed duck } \\
\text { Anas erythrorhynchas }\end{array}$ & + & + & Uncommon & Uncommon \\
\hline 7. & $\begin{array}{l}\text { White- backed vulture } \\
\text { Gyps bengalensis }\end{array}$ & - & + & Uncommon & Rare \\
\hline 8. & $\begin{array}{c}\text { Lammergeyer } \\
\text { Gypaetus barbatus }\end{array}$ & - & + & - & Rare \\
\hline 9. & $\begin{array}{l}\text { African fish Eagle } \\
\text { Halieetus vocifer }\end{array}$ & - & + & Rare & Rare \\
\hline 10. & $\begin{array}{l}\text { Long-crested Eagle Lophaetus } \\
\text { occipitalis }\end{array}$ & - & + & - & Rare \\
\hline 11. & $\begin{array}{l}\text { Augur buzzard } \\
\text { Buteo rufofuscus }\end{array}$ & - & + & - & Fairly Common \\
\hline 12. & $\begin{array}{c}\text { Back kite } \\
\text { Milvus migrans }\end{array}$ & + & + & Fairly Common & Fairly Common \\
\hline 13. & $\begin{array}{l}\text { Verreax's Eagle } \\
\text { Aquila verreavxii }\end{array}$ & + & - & & - \\
\hline 14. & $\begin{array}{c}\text { Martial Eagle } \\
\text { Polemeatus bellicosus }\end{array}$ & + & - & Rare & - \\
\hline 15. & $\begin{array}{c}\text { Crowned Eagle } \\
\text { Stephanoaetus caronnatus }\end{array}$ & + & - & Rare & - \\
\hline 16. & $\begin{array}{l}\text { Grasshopper buzzard } \\
\text { Butastus rufipennis }\end{array}$ & + & - & Uncommon & - \\
\hline 17. & $\begin{array}{c}\text { Lizard byzzard } \\
\text { Kaupifalco monogrammicus }\end{array}$ & + & - & Rare & - \\
\hline 18. & $\begin{array}{l}\text { Red-necked spurfowl } \\
\text { Francolinus levcoscepus }\end{array}$ & + & - & Uncommon & - \\
\hline 19. & $\begin{array}{l}\text { Helmeted guineafowl numida } \\
\text { meleagril }\end{array}$ & + & - & Fairly Common & \\
\hline 20. & $\begin{array}{c}\text { Scaly francolin } \\
\text { Francolinus squamatus }\end{array}$ & + & - & Uncommon & - \\
\hline 21. & $\begin{array}{c}\text { Red-eyed dove } \\
\text { Sreptopeha semitorquata }\end{array}$ & + & - & Fairly Common & - \\
\hline 22. & $\begin{array}{l}\text { Ring-necked dove } \\
\text { Streptopeha capicola }\end{array}$ & + & + & Fairly Common & Rare \\
\hline
\end{tabular}




\section{Journal of Ecology and Natural Resources}

\begin{tabular}{|c|c|c|c|c|c|}
\hline 23. & $\begin{array}{l}\text { Green pigeon } \\
\text { Treron olax }\end{array}$ & + & + & Uncommon & Rare \\
\hline 24. & $\begin{array}{c}\text { Laughing dove } \\
\text { Streptopeha senegalensis }\end{array}$ & + & - & Fairly Common & - \\
\hline 25. & $\begin{array}{l}\text { Blue-naned mousebird colius } \\
\text { macrourus }\end{array}$ & - & + & - & Rare \\
\hline 26. & $\begin{array}{c}\text { Speckled mousebird } \\
\text { Colius striatus }\end{array}$ & - & + & - & Rare \\
\hline 27. & $\begin{array}{l}\text { Emerald cuckoo } \\
\text { Chrsococcyx cupreus }\end{array}$ & + & + & Rare & Rare \\
\hline 28. & $\begin{array}{l}\text { Red-chested cuckoo } \\
\text { Cuculus solitarrius }\end{array}$ & + & + & Uncommon & Rare \\
\hline 29. & $\begin{array}{l}\text { White-braved coucal centropus } \\
\text { superciliosus }\end{array}$ & + & + & Uncommon & Rare \\
\hline 30. & $\begin{array}{l}\text { Great blue turaco } \\
\text { Corythaeola cristat }\end{array}$ & - & + & - & Rare \\
\hline 31. & $\begin{array}{c}\text { Wattled black hornbill ceratogyma } \\
\text { atrata }\end{array}$ & + & + & Uncommon & Rare \\
\hline 32. & $\begin{array}{l}\text { White-crested Hornbill Tropicranus } \\
\text { albcristatu }\end{array}$ & + & + & Uncommon & Rare \\
\hline 33. & $\begin{array}{c}\text { Grey Hornbill } \\
\text { Tockus hemprichii }\end{array}$ & + & + & Uncommon & Rare \\
\hline 34. & $\begin{array}{l}\text { Yellow-billed Hornbill Tockus } \\
\text { flavirossris }\end{array}$ & + & + & Uncommon & Rare \\
\hline 35. & $\begin{array}{c}\text { Golden pipit } \\
\text { Tmetothylacus tenellus } \\
\end{array}$ & + & - & Uncommon & - \\
\hline 36. & $\begin{array}{l}\text { Yellow-throated long claw } \\
\text { Macronyx croceus }\end{array}$ & + & - & Rare & - \\
\hline 37. & $\begin{array}{l}\text { Singing cisticola } \\
\text { Cisticola cantans } \\
\end{array}$ & + & - & Rare & - \\
\hline 38. & $\begin{array}{c}\text { Crombec } \\
\text { Sylivietta brahyura }\end{array}$ & + & - & Rare & - \\
\hline 39. & $\begin{array}{c}\text { Back and white manikin } \\
\text { Lonchura poensis }\end{array}$ & + & - & Rare & - \\
\hline 40. & $\begin{array}{c}\text { Bronze manikin } \\
\text { Lonchura cucullata }\end{array}$ & + & - & Rare & - \\
\hline 41. & $\begin{array}{l}\text { Rufous-backed manikin } \\
\text { Loncura nigriceps } \\
\end{array}$ & + & - & Fairly Common & - \\
\hline 42. & $\begin{array}{l}\text { Hunter's sunbird } \\
\text { Nectarinca hunter }\end{array}$ & + & - & Uncommon & - \\
\hline 43. & $\begin{array}{l}\text { Green-throated sunbird } \\
\text { Nectarinia rubescens }\end{array}$ & + & - & Rare & - \\
\hline 44. & $\begin{array}{l}\text { Olive-bellied sunbird } \\
\text { Nectarinia choloropygius }\end{array}$ & + & - & Fairly Common & - \\
\hline 45. & $\begin{array}{l}\text { Red-chested sunbird } \\
\text { Nectarinea erythrocera }\end{array}$ & + & - & Uncommon & - \\
\hline 46. & $\begin{array}{l}\text { Regal sunbird } \\
\text { Nectarinia ronia }\end{array}$ & + & - & Uncommon & \\
\hline
\end{tabular}




\section{Journal of Ecology and Natural Resources}

\begin{tabular}{|c|c|c|c|c|c|}
\hline 47. & $\begin{array}{l}\text { Scarlet-tufted malachite sunbird } \\
\text { Nectarinia johnstoni }\end{array}$ & + & - & Uncommon & - \\
\hline 48. & $\begin{array}{l}\text { Malachite sunbird } \\
\text { Nectarinia famosa }\end{array}$ & + & - & Uncommon & - \\
\hline 49. & $\begin{array}{l}\text { Purple-breasted sunbird } \\
\text { Nectarinia purpureventris }\end{array}$ & + & - & Rare & - \\
\hline 50. & $\begin{array}{l}\text { Shinning sunbird } \\
\text { Turtur hypanistria }\end{array}$ & + & - & Rare & \\
\hline 51. & $\begin{array}{l}\text { Tambourine dove } \\
\text { Turtur tympanistria }\end{array}$ & + & + & Uncommon & Rare \\
\hline 52. & $\begin{array}{l}\text { Green pigeon } \\
\text { Treon australis }\end{array}$ & + & + & Uncommon & Rare \\
\hline 53. & $\begin{array}{l}\text { Great white Egret } \\
\text { Egretta alba }\end{array}$ & + & - & Fairly common & - \\
\hline 54. & $\begin{array}{l}\text { Emerald-spptted wood dove } \\
\text { Turtur chalcospilos }\end{array}$ & + & - & Uncommon & - \\
\hline 55. & $\begin{array}{c}\text { Little bittern } \\
\text { Ardeirallus sturmii }\end{array}$ & - & + & - & Rare \\
\hline 56. & $\begin{array}{l}\text { African hobby } \\
\text { Falco cuvieri }\end{array}$ & - & + & - & Rare \\
\hline 57. & $\begin{array}{l}\text { Yellow-necked spurfowl } \\
\text { Francolinus leucoscepus }\end{array}$ & + & + & Rare & Rare \\
\hline 58. & $\begin{array}{l}\text { Speckled pigeon } \\
\text { Columba guinea }\end{array}$ & + & + & Uncommon & Rare \\
\hline 59. & $\begin{array}{l}\text { Black Hornbill } \\
\text { Ceratoauma atrata }\end{array}$ & - & + & - & Uncommon \\
\hline
\end{tabular}

Table 4: Seasonal occurrence of bird species observed in disturbed and undisturbed vegetation of Ikere Forest Reserve.

\section{Discussion}

Species richness is often used to make quick assessment and comparison of different habitats and may reveal interesting general pattern which is very essential to understand the health of the ecosystems and to develop efficient and effective sustainable bird conservation strategies and management measures. Species abundance and diversity are therefore useful considerations when assessing bird community. The species abundance and diversity in the disturbed and undisturbed vegetation of Ikere Forest Reserve are markedly different. The structurally less complex vegetation of the disturbed habitat had higher diversity than the more complex vegetation of the undisturbed study area. Also the bird species abundance differs between the disturbed and undisturbed vegetation of the reserve. The reason that may be ascribed for this variation may be as a result of farming practices which result in more distinct difference in the vegetation cover between the two vegetation types. The difference in bird species abundance and diversity in the two vegetation types may therefore reflect this difference.
In this study, order Passeriformes was numerically the dominant order represented with 15 species from the 59 identified species which accounts $32.0 \%$ of the identified species.This result is in agreement with Tesfahonegy [4] in Kafa biosphere reserve, southwest Ethiopia which reported that among the 42 species identified, $47.6 \%$ of the species belonged to the order Passeriformes. The present study in Ikere Forest Reserve, vividly showed that the disturbed vegetation support a higher number of bird species (78\%) and bird abundance (71.3\%) as compare to undisturbed vegetation of the reserve. This is in line with the result of Aynalem and Bekele [12], Mengesha [13] where high species diversity, richness and abundance of birds was observed in the disturbed vegetation and which might be associated with the difference in the vegetation community structure of the two sites that determine food, water, cover availability, nestling and protection opportunities based upon the bird's habitat preference and feeding habits. The effects of anthropogenic disturbance on avian communities was reported by Lambert [14] and John [15] to include increase species richness due to increase in the presence of forest edge species, but a reduction in the relative abundance of certain 
species or groups of species. The record of 59 bird species during this study shows that bird species diversity in the vegetation types of the reserve was relatively low. Also the bird species community was dominated by few species, most of which had low frequency of occurrence. The vegetation types also comprised wide ranges of resident bird species. Similar results were found by Mengesha [13] that disturbed vegetation of the Abijata Shalla Lakes National Park, Ethiopia formed resources areas for resident birds. The variation in bird species composition and abundance among the two vegetation types may be attributed to more of homogeneous plant species present in the undisturbed vegetation and high densities of heterogeneous flora in the disturbed vegetation which invariably bring about the variation in food, cover availability, micro-climatic variation and predation pressure. Afolayan and Ajayi [16] and Crowel [17] had observed that the distribution of fauna species is largely dependent on the availability of food, water, and cover. The disturbed vegetation of the reserve that supports the larger number of species comprises of diverse flowering and agricultural plants whose fruits might be responsible for high number of bird species recorded. The relative abundance estimates of birds differed in values and the differences are dependent on vegetation structures. Thus, the disturbed vegetation contained more bird species, order and families than the undisturbed vegetation. In contrast, Wilbard [18] has reported higher avian species, order, family and diversity was found in the disturbed vegetation of Kilombero wetland, Tanzania and ascribed the variation to plant species and feeding habits of the observed bird species. Cody [19]; Igi and Ballard [20] noted that the relative abundance of bird species was higher in habitats dominated by woody vegetation or habitats that are structurally and floristically more diverse. Also, the structural and complexity of habitat and diversity of vegetation forms showed significant correlations with fauna species diversity [21]. The higher abundance of individual birds in the disturbed vegetation could also be attributed to the composition of vegetation that forms the main element of the habitat Lee and Rotenberry [22]; Chapman and Reich [23] whereas the undisturbed vegetation is fairly homogenous and so has an even nature of vegetation. The differences between the numbers of birds recorded in the vegetation types were not significant. Species richness and diversity indices of disturbed and undisturbed vegetation suggest that the bird species observed are different in terms of composition. The disturbed and undisturbed vegetation types showed fairly pronounced number of shared bird species (18 species) because these vegetation types had many plant species in common. However, the disturbed vegetation type had higher human interferences and the ensuing colonization has diversified the food resources available to bird species of different feeding guilds. The highest numbers of rare bird species occurring in the undisturbed vegetation is typical of such vegetation [24]. Giller [25] had noted that historical and ecological factors influences species richness in a habitat. The identified historical factors are mainly speciation and supply of colonists while the ecological factor relates to mortality from predation by the birds of prey [26]. The higher index of dominance recorded in the undisturbed vegetation is due to more of bird species that had higher relative abundance values. The dominance of these bird species revealed their survival superiority over other species and the high encounter rates, presence of favourable habitats for certain bird species and high defense against predations [27].

\section{Conclusion}

Birds are critically important component of an ecosystem as they act as bio-monitors, alerting people to what is going wrong in an environment. Birds can also be referred to as habitat quality indicators due to the fact that its population decline when habitat quality is very poor. Therefore, it is necessary to have a data base for the diversity and distribution of bird species present in the disturbed and undisturbed vegetation types of Ikere Forest Reserve which have high ornithological value in view of the presence diverse bird species inhabiting the area.

From the present study it is revealed that the bird species composition and abundance varies in the two study vegetation types. Bird species composition and abundance was higher in the disturbed vegetation related to high densities of heterogeneous flora which bring about variation in food, cover availability, micro-climatic variation and predation pressure. The disturbed vegetation did not alter species richness and abundance negatively but did alter the identities and relative abundance of avian species by supporting more bird species than the undisturbed vegetation. In Ikere Forest Reserve, Ekiti State, vegetation types both primary and secondary play important roles in the survival and distribution of bird species it supports. If a substantial part of the bird community in the reserve is to be maintained, effort must be geared toward conservation of both the disturbed and undisturbed vegetation types of the reserve. This study suggests that a landscape of both the disturbed and undisturbed vegetation types would support birdlife. The occurrence of higher diversity and abundance of birds in the disturbed vegetation indicate that the vegetation type is still within tolerable level or in reasonable ecological conditions.

\section{References}

1. Elgood JH (1982) Birds of Nigeria. B.O.U. Pub; England, pp: 246.

2. Ogunyemi OO (2012) Ecological Diversity and abundance 
of birds in the wetlands of Ekiti State. unpublished Ph.D Thesis in the Department of Ecotourism and Wildlife Management, Federal University of Technology, Akure (FUTA), Nigeria.

3. Watson JEM, Whittaker RJ, Dawson TP (2005) The importance of Littoral forest remnants for indigenous bird conservation in southeastern Madagascar. Biodiversity Conservation 14: 523-545.

4. Tesahuneguy W, Fekensa T, Mulualem G (2016) Avifauna Diversity in Kafa Biosphere Reserve: Knowledge and Perception of Villagers in Southwest Ethiopia. Ecology and Evolutionary Biology 1(2): 7-13.

5. Rands MRW, Adams WM, Bennun L, Butchar SHM, Clements A, et al. (2010) Biodiversity conservation: Challenges beyond 2010. Science 329(5997): 12981303.

6. Leito A, Truu J, Roosaluste E, Seep K, Podier I (2006) Long term dynamics of breeding birds in broad-leaved deciduous forest on Hanckasti Island in the WestEstonian archipelago. Ornis Fennica 83: 124-130.

7. Wiens DA (1989) The Ecology of Bird Communities, Cambridge University Press, Cambridge, pp: 539.

8. Sethy J, Sama D, Sethi S (2015) Species diversity and abundance of birds in and around North Orissa University, Takatpur, Baripada, Mayurbhanj, Odisha. Int J innovative Res Sci Eng Technol 4(2): 1-12.

9. Bibby CJ, Burgess ND, Hill DA, Mustoe SH (1998) Bird Census techniques. Academic Press, London.

10. Jones M (1998) Study design. In: Bibby C, Jones M, Marseden S (Eds.) Expedition field techniques, Birds surveys. Royal Geographical Society with the institute of British Geographer, London, pp: 15-34.

11. Oksanen L (2001) Logic of experiments in ecology: Is pseudoreplication a pseudoissue? Oikos 94(1): 27-38.

12. Aynalem S, Bekele A (2008) Species composition, relative abundance and distribution of bird fauna of riverine and wetland habitats of Infranz and Yiganda at southern tip of Lake Tana, Ethiopia. Tropical Ecology 49(2): 199-209.

13. Mengesha G, Mamo Y, Bekele A (2015) A comparison of terrestrial bird community structure on bird assemblages of oak (Quercus ssp.) and pine (Pinus ssp.) stands in Southwestern Turkey. Forest Ecology and Management 336: 137-147.

14. Lambert FR (1992) The consequences of selective logging for Bornean lowland forest birds. Phils. Trans R.Soc Lond 335(1275): 443-457.
15. John AG (1995) Bird population persistence in sabahan logging concessions. Biological Conservation 75(1): 3-10.

16. Afolayan TA (1980) The influence of seasonality on the distribution of large mammals Yankari; Game Reserve, Nigeria. African Journal of ecology 18(1): 87-93.

17. Crowel M, Schif JC, Gubb AA (1981) Effect of rainfall variation, fire vegetation and habitat physiognomy on Northern Animal community. South Africa Wildlife Resource 11(3): 87-104.

18. Wilbard AN, Samora MA (2013) Bird species composition and diversity in habitats with different disturbance histories at Kilombero wetland, Tanzania. Journal of Ecology 3(7): 482-488.

19. Cody MC (1985) Habitat selection in Birds. Academic press Inc, California.

20. Igi LO, Ballard BM (1999) Habitat associations of migrating and overwintering grassland birds of southern Texas. Condor 101(4): 771-782.

21. Girma Z, Mengesha G, Asfaw T (2017) Diversity, relative abundance of avian fauna in and around Wondo Genet Forest, South-central Ethiopia. Research Forestry 11(1): 1-12.

22. Lee PY, Rotenberry JT (2005) Relationships between bird species and tree species assemblages in forested habitats of eastern North America. Biogeogr J 32(7): 1139-1150.

23. Champan KA, Reich PB (2007) Land use and habitat gradients determine bird community diversity and abundance in suburban, rural and reserve landscapes of Minnesota, U.S.A. Biological Conservation 135(4): 527 541.

24. Lovejoy TE (1975) Bird diversity and abundance in Amazon forest communities. Living Bird 13: 127-191.

25. Giller PS (1984) Community structure and the Niche, Chapman and Hall: 58.

26. Shannon CE, Weaver W (1949) The mathematical Theory of combination, University of Illinois press, Urbana Illinois.

27. Sorensen TA (1948) A method of establishing group of equal amplitude in plant sociology based on similarity of species content, and its application to analyses of the vegetation on Danish commons. $\mathrm{K}$ dan vidensk Slsk Bio Skr 5: 1-34.

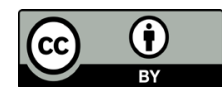

